\title{
Why Cochrane should prioritise sharing data
}

In this Letter (BMJ 2018;362:k3229, doi:10.1136/bmj.k3229), Clive E Adams was listed with the wrong affiliation. The correct affiliation is Institute of Mental Health, University of Nottingham, Nottingham, UK. 